\title{
A Study on Silk Fabric Applied with Eco- Friendlycolorants for the Enhancement of Textile Properties
}

\author{
Sathish, $\mathrm{P}^{1}$ and Jeyakodi Moses, $\mathrm{J}^{2 *}$ \\ \{atoms2020@gmail.com ${ }^{1}$, jjmoses2k2@gmail.com ${ }^{2}$ \} \\ ${ }^{1}$ Ph.D. Research Scholar, Research \& Development Centre, Bharathiar University, Coimbatore \\ 641046, India. ORCID ID : 0000-0002-2885-3203, ${ }^{2}$ Associate Professor, Department of Applied \\ Science, PSG College of Technology, Coimbatore 641004, India. ORCID ID : 0000-0002-9530-8731
}

\begin{abstract}
Silk is possessed with valuable textile materials of unique properties like sheen, softness, luster, elegance, strength, uniformity make it suitable for the production of unique textile and garment products.Compared to other textiles like cotton; silk consists of the functional groups in different aspects suitable in the application of both the acid and the basic mild conditions. Hence, silk could be subjected with the coloration process using various dye categories. With respect to this, it is proposed in the present work to increase the characteristics of domestic mulberry silk fabric by treating with collected sericinalong with natural finishing sources like aloevera, amla and nochi in varied combinations followed by coloring process using somespecific natural sources. The silk fabrics were then subjected for some specific testing process for measuring the coloration data, fastness characters, hand values, absorption criteria, uv protection aspects, $x$ ray diffraction micrographs and scanning electron microscope images. The results received are in the satisfaction level suitable for theproduction of textile products especially garment and apparels.
\end{abstract}

Keywords: Silk Fabricm, Eco Friendlycolorants, Textile.

\section{Introduction}

The two families which spin silk fiber are Saturnidae and Bombycidae. Bombyx mori which belongs to Bombycidae produces the soft and delicate dual threads of protein called fibroin. The protein in silk is an essential constituent of the filament possessed in cocoon similar to that of the keratin, collagen, sporgin, and elastin [1-3]. The silk comprised of fiber protein is synthesized by the gland cells stored in the lumen. The silk fibers constitute protein $97 \%$, in that fibroin is a fiber protein in filament form and sericin - a gum is considered as non fibrous protein. In addition, other unlike components such as carbohydrates, wax, inorganic salts and pigments are also present [3-5]. Sericin is insoluble in cold water but easily hydrolyzed in an alkaline medium. The presence of sericin in silk fiber increases the strength, reduces the luster and hence in order to enhance the luster the raw silk must be degummed prior to dyeing [4,6]. Silk filament and fiber find use in many products like industrial fabrics, medical sutures, aero-parachutes, tire-lining materials, and also artificial-blood vessels [5, 7].

The softness, the translucence, as well as the smoothness revealed by the purified silk is with regard to their varied extents of intermittent transmittance, smooth surface reflections 
as well the diffused and reflected light. Many studies of the research workers by use of X-ray techniques on silk related the properties namely handle, luster, brightness with its crystal structure $[6,8,9]$. In spite of all these inherent properties of silk, in this research work it is proposed to treat the raw mulberry silk fabric for degumming to collect sericin which was used for the application along with selected natural sources such as aloevera, amla and nochi in different combinations followed by the coloration with some listed natural sources like that of the Madder, Red Sandal Wood, Barberry, Annatto, Myrobalan, Pomogranate, Onion, and Grape. Finally, from this research work, the results received were convincing corresponding to the possibility of utility in the textile and garment product purposes.

\section{Materials \& Methods}

\subsection{Materials}

The raw mulberry silk fabric - $100 \%$, Woven, plain - was collected from the Gandhipuram Sharvodaya Sangh (Vadavalli, Coimbatore, Tamil Nadu, India). The specifications involved for the raw silk fabric are as: warp and weft count 2 / 80's respectively, ends / inch 100, picks / inch 60, G S M 95, and cloth width 44 inches.

The original natural resources, namely; 1) madder, 2) red sandal wood, 3) barberry, 4) annatto, 5) myrobalan, 6) pomogranate, 7) onion, and 8) grape as well as I) Aloevera, II) Amla, and III) Nochi are collected from the Forest Department (Coimbatore, Tamil Nadu, India).

The selected original natural sources; Madder, Red Sandal Wood, Barberry, Annatto, Myrobalan, Pomogranate, Onion, and Grape are utilized for the purpose of coloration. Whereas, Aloevera, Amla, and Nochi are involved for improving the functional application purposes in addition to the recovered Sericin (Protein) obtained from the raw silk fabric; individually as well as in the combination of them over the silk fabric with the coloring natural sources.

The selected chemicals \& auxiliaries noted in this study elsewhere for the treatments are purchased in the $\mathrm{A} R$ grade.

\subsection{Methods}

\section{2a. Raw mulberry silk fabric treatment}

A treatment of 10-gpl $\mathrm{HCl}$ was applied on the mulberry (raw) silk fabric for sixty minutes in the respective baths separately with the material to liquor ratio-MLR of $1: 30$ at around $40^{\circ} \mathrm{C}$ for the removal of the natural and added unwanted-impurities. The process of degumming was then carried out by sodium carbonate $2 \%$ on weight of material (owm) \& commercial soap powder for degumming $(2 \% \mathrm{owm})$ at around $85^{\circ} \mathrm{C}$ for nearly two hours. Finally, the silk fabric was washed thoroughly using de-ionized water and dried completely [10-12].

\section{2b. Collection of sericin from the degum bath}

The degumming solution was collected safely, preserved and then subjected for the purification in order to recover the sericin, as per the established technique $[13,14]$. 


\section{2c. Process of extraction from natural sources}

The extraction process as preferred by the established standard methods were incorporated on the selected natural sources namely Madder, Red Sandal Wood, Barberry, Annatto, Myrobalan, Pomogranate, Onion, Grape, Aloevera, Amla, and Nochi [15,16].

\section{2d. Process of coloration and K/S value determination}

The natural sources extracted for coloration were applied on the silk fabrics, which were treated already with the functional category of natural sources (aloevera, amla, nochi and extracted protein) in differed aspects for the improvement of natural coloration and finishing values in the following manner:

0 - without any treatment ; a - with recovered protein ; b- aloevera with protein

c- amla with protein $\mathrm{d}$ - nochi with protein

A - Protein with (aloevera and amla); B - Protein with (Aloevera and Nochi) CProtein with (Amla and Nochi); D - Protein with (Aloevera, Amla and Nochi)

The silk fabrics were colored by the established standard technique with the extracted natural sources of the concentration of 25 gram per litre at nearly boil temperature for two around hours with the MLR of 1:30. The color strength value (K/S) was then calculated with the help of Kubelka-Munk relation, represented as; $\mathrm{K} / \mathrm{S}=\left((1-\mathrm{R})^{2} / 2 \mathrm{R}\right)[17-21]$.

\section{2e. Measuring the fastness property values on the natural colored silk fabrics}

The natural colored silk fabrics were subjected with established standard AATCC test methods for the measurement of fastness properties (like wash, light, and rubbing) based on the techniques of $61-1996,2003 ; 16-1998,2003 ; 8-2007,2007 ; 135-1985,2003$ respectively [22-25].

\section{KES-F}

2.2f. Measurement of the objective assessment on the natural colored silk fabrics by

The hand properties with respect to the mechanical and surface properties of the natural colored silk fabrics were measured by the Kawabata evaluation system (KES-F) [26].

2.2g. Measurement of the air and water vapor permeability of natural colored silk fabrics

The ASTM D737 was used for measuring the air permeability; and the ASTM E96 moisture vapor test was used for measuring the WVP rate of the natural colored silk fabrics $[27,28]$.

2.2h. Measurement of the UPF assessment in the natural colored silk fabrics

According to the standard established method, the silk fabric was subjected with uv protection finishing (Super FX Anti UV) and tested based on the requirement $[29,30]$.

\section{2i. Test for the XRD of natural colored silk fabrics}

In the natural colored silk fabrics, the unknown crystalline materials present were determined using XRD (Shimadzu XRD6000) technique [31,32]. 


\section{2j Test for the SEM of the natural colored silk fabrics}

The colored silk fabrics were undergone for the scanning-electron-microscope study using the 30-kV scanning-electron-microscope (JEOL-Japan, Model J S M - 6360) [33,34].

\section{Results And Discussion}

\subsection{Colorimetric and color fastness data of the natural colored silk 3.1a. Color value of the natural colored silk}

The color values of the natural sources such as; Madder, Red Sandal Wood, Barberry, Annatto, Myrobalan, Pomegranate, Onion, and Grape, natural colored on silk fabric applied with sericin (protein), aloevera, amla, nochi in different combinations are shown in the Table 1a. It is noted from the Table 1a that the silk fabric gets good colors in the manner of Red, Orange-Red, Yellow, Orange, Green, Brown, Red Orange and Purple as colored using the help of natural sources such as Madder, Red Sandal Wood, Barberry, Annatto, Myrobalan, Pomogranate, Onion, and Grape. It is also observed from this table, the treated $(0, a, b, c, d)$ silk fabrics dyed with natural sources show the least colorimetric data compared with those of the other treated (A, B, C, D) silk fabrics. Sericin - protein was applied on silk fabrics with aloevera, amla, nochi individually as well as in combination with the each other. It is evidenced that, among all these treated silk fabrics, the colorimetric data is maximum for those applied with sericin with aloevera, amla and nochi and least in the case of sericin treated only fabrics. From the Table 1a, it is evident that the presence of aloevera enhances the colorimetric data considerably. In the silk fabric treated with aloevera and nochi along with the protein, it is seen that there is a remarkable increase in the colorimetric data.

Kubelka-Munk theory is used as the most common approach to the optical properties of the substrate and colorant. As the opaque material surfaces are exposed to the light then the reflection, absorption and scattering occur. The reflectance shown by the colored textile substrate as the value of an absorption-spectrum, $K(\lambda)$ and a scattering-spectrum of the textile substrate $S(\lambda)$ is described by the source light interacting with a textile fiber substrate which reveals the absorption/scattering of light by the respective colored molecules and the textile fibers. Hence, the behavior of a dye can be predicted at a given concentration, due to the fact that the colorimetric data can be found at various wavelengths.

\section{1b. Color fastness data of the natural colored silk}

The color fastness values like wash, light, and rub fastness properties of natural colored silk fabrics applied with sericin, aloevera, amla and nochi in different combinations are also shown in Table 1b. It is noted from the table that the overall fastness properties are maximum for the silk fabrics with all the combined treatment, however they are least for the natural colored silk fabrics without any treatment. There is moderate to good trend in the general fastness properties on all the treated and natural colored silk fabrics, but still in all the cases, the wet rubbing fastness is considerably less. The fastness property values exhibited by the silk fabrics natural colored with annatto, myrobalan and pomegranate are relatively good. AATCC defines the color fastness as the resistive force of a textile fiber substrate to change in any of the color characters, to transfer the colorant(s) to the nearby textile substrates, or in 
both, with regard to the exposure of the textile material to any reliable condition which may be met in the textile processing, testing, storage, or in the use of textile products. In general, it is mentioned as the capability to contain the color throughout the life cycle. Also, in textile testing, varied types of colorfastness characters that are assumed to give the end users with an anticipated product.

Table 1a. Colors obtained and colorimetric data on the natural colored silk

\begin{tabular}{|c|c|c|c|c|c|c|c|c|c|c|c|}
\hline \multicolumn{3}{|c|}{$\begin{array}{l}\text { Natural sources \& } \\
\text { Colors obtained }\end{array}$} & \multicolumn{9}{|c|}{ Color values } \\
\hline $\begin{array}{l}\text { Natural } \\
\text { Sources }\end{array}$ & $\begin{array}{l}\text { Botanical } \\
\text { Name }\end{array}$ & $\begin{array}{l}\text { Colors } \\
\text { Obtained }\end{array}$ & $\mathbf{0}$ & $\mathbf{a}$ & b & c & d & $\mathbf{A}$ & B & $\mathbf{C}$ & D \\
\hline i) Madder & $\begin{array}{l}\text { (Rubiacardi } \\
\text { folia) }\end{array}$ & Red & 12.7 & 12.8 & 13.3 & 13.0 & 13.2 & 13.5 & 13.4 & 13.4 & 13.6 \\
\hline ii) RS Wood & $\begin{array}{l}\text { (Pterocarpus } \\
\text { santallinus) }\end{array}$ & Orange red & 12.6 & 12.9 & 13.4 & 13.1 & 13.3 & 13.6 & 13.7 & 13.5 & 13.8 \\
\hline iii) Barberry & $\begin{array}{l}\text { (Berberis } \\
\text { vulgaris) }\end{array}$ & Yellow & 12.3 & 12.9 & 13.5 & 13.2 & 13.3 & 13.6 & 13.7 & 13.6 & 13.8 \\
\hline iv) Annatto & $\begin{array}{l}\text { (Bixa } \\
\text { orellana) }\end{array}$ & Orange & 13.0 & 13.1 & 13.5 & 13.2 & 13.4 & 13.7 & 13.7 & 13.6 & 13.9 \\
\hline v) Myrobalan & $\begin{array}{l}\text { (Terminalia } \\
\text { chebula) }\end{array}$ & Green & 12.4 & 12.7 & 13.3 & 12.9 & 13.1 & 13.6 & 13.7 & 13.5 & 13.8 \\
\hline $\begin{array}{l}\text { vi) } \\
\text { Pomogranate }\end{array}$ & $\begin{array}{l}\text { (Punica } \\
\text { granatumm) }\end{array}$ & Brown & 12.9 & 13.2 & 13.6 & 13.3 & 13.5 & 13.7 & 13.8 & 13.7 & 13.9 \\
\hline vii) Onion & $\begin{array}{l}\text { (Allium } \\
\text { cepa) }\end{array}$ & Red orange & 12.8 & 12.9 & 13.4 & 13.1 & 13.3 & 13.6 & 13.7 & 13.5 & 13.8 \\
\hline viii) Grape & $\begin{array}{l}\text { (Citrus } \\
\text { paradise) }\end{array}$ & Purple & 12.5 & 12.7 & 13.4 & 12.9 & 13.1 & 13.5 & 13.7 & 13.4 & 13.8 \\
\hline \multicolumn{3}{|l|}{ Mean } & 12.7 & 12.9 & 12.7 & 12.9 & 13.4 & 13.1 & 13.3 & 13.6 & 13.7 \\
\hline \multicolumn{3}{|c|}{ Standard Deviation } & 0.19 & 0.17 & 0.19 & 0.17 & 0.09 & 0.14 & 0.13 & 0.07 & 0.11 \\
\hline \multicolumn{3}{|l|}{ Variance } & 0.04 & 0.03 & 0.04 & 0.03 & 0.01 & 0.02 & 0.02 & 0.01 & 0.01 \\
\hline
\end{tabular}

RS Wood Red Sandal Wood

0 - without any treatment ; a - with recovered protein ; b- aloevera with protein

c- amla with protein $\mathrm{d}$ - nochi with protein

A - Protein with (aloevera and amla); B - Protein with (Aloevera and Nochi) CProtein with (Amla and Nochi); D - Protein with (Aloevera, Amla and Nochi)

Table 1b. Color fastness data of the natural colored silk

\begin{tabular}{|c|c|c|c|c|c|c|c|c|c|c|c|c|c|c|c|c|c|c|}
\hline $\begin{array}{c}\text { S. } \\
\text { No. }\end{array}$ & \multicolumn{9}{|c|}{ Wash fastness } & \multicolumn{9}{|c|}{ Light fastness } \\
\hline No. & \begin{tabular}{|c}
$\mathbf{0}$ \\
$2-$ \\
3
\end{tabular} & $\begin{array}{l}\mathbf{a} \\
3\end{array}$ & \begin{tabular}{|l}
$\mathbf{b}$ \\
$3-4$
\end{tabular} & $\begin{array}{l}\text { c } \\
3-4\end{array}$ & \begin{tabular}{|l} 
d \\
$3-4$
\end{tabular} & $\begin{array}{l}\text { A } \\
3-4\end{array}$ & \begin{tabular}{|l} 
B \\
$3-4$
\end{tabular} & $\begin{array}{l}\mathbf{C} \\
3\end{array}$ & \begin{tabular}{|l|} 
D \\
$3-4$
\end{tabular} & $\begin{array}{l}\mathbf{0} \\
3-4\end{array}$ & $\begin{array}{l}\mathbf{a} \\
3-4\end{array}$ & $\begin{array}{l}\text { b } \\
4\end{array}$ & $\begin{array}{l}\text { c } \\
4\end{array}$ & $\begin{array}{l}\text { d } \\
4\end{array}$ & $\begin{array}{l}\text { A } \\
4\end{array}$ & $\begin{array}{l}\text { B } \\
4\end{array}$ & $\begin{array}{l}\mathbf{C} \\
4\end{array}$ & $\begin{array}{l}\mathbf{D} \\
4-5\end{array}$ \\
\hline ii & $\begin{array}{c}2- \\
3\end{array}$ & 3 & 3 & 3 & 3 & 3 & 3 & 3 & $3-4$ & 3 & $3-4$ & $3-4$ & $3-4$ & $3-4$ & $3-4$ & 4 & $3-4$ & 4 \\
\hline
\end{tabular}




\begin{tabular}{|c|c|c|c|c|c|c|c|c|c|c|c|c|c|c|c|c|c|c|}
\hline iii & 3 & 3 & 3-4 & 3 & 3 & 3 & 3-4 & 3 & 3-4 & 3-4 & 4 & 4 & 4 & 4 & 4 & 4 & 4 & 4 \\
\hline iv & $\begin{array}{c}2- \\
3\end{array}$ & 3 & 3-4 & 3 & 3-4 & 3-4 & $3-4$ & 3-4 & 4 & 3-4 & $3-4$ & 4 & 3-4 & 4 & 4 & 4-5 & 4 & $4-5$ \\
\hline V & 3 & 3-4 & 3-4 & 3-4 & 3-4 & 3-4 & 3-4 & 3-4 & 3-4 & 3-4 & 4 & 4 & 4 & 4 & 4 & 4 & 3-4 & 4-5 \\
\hline vi & 3 & 3-4 & 3-4 & 3-4 & 3-4 & 3-4 & 3-4 & 3-4 & 4 & 3-4 & 3-4 & 4 & 4 & 4 & 4 & 4-5 & 4 & $4-5$ \\
\hline vii & 3 & 3 & 3 & 3 & 3 & 3 & 3 & 3 & 3-4 & 3 & 3-4 & 4 & 3-4 & 3-4 & 4 & 4 & $3-4$ & 4 \\
\hline viii & $\begin{array}{c}2- \\
3\end{array}$ & 3 & 3 & 3 & 3 & 3 & 3 & 3 & 3 & 3 & 3-4 & 3-4 & 3-4 & 3-4 & $3-4$ & 3-4 & 3-4 & 4 \\
\hline$M$ & $\begin{array}{c}2- \\
3\end{array}$ & 3 & 3-4 & 3 & 3 & 3 & 3 & 3 & 3-4 & 3 & $3-4$ & 3-4 & $3-4$ & 3-4 & 3-4 & 4 & $3-4$ & 4 \\
\hline$S D$ & 0.4 & 0.4 & 0.5 & 0.4 & 0.5 & 0.5 & 0.5 & 0.5 & 0.5 & 0.5 & 0.5 & 0.4 & 0.5 & 0.4 & 0.4 & 0.4 & 0.5 & 0.5 \\
\hline$V$ & 0.1 & 0.1 & 0.3 & 0.1 & 0.2 & 0.3 & 0.3 & 0.2 & 0.3 & 0.3 & 0.3 & 0.1 & 0.2 & 0.1 & 0.1 & 0.1 & 0.2 & 0.3 \\
\hline
\end{tabular}

S. Rub fastness

\begin{tabular}{|c|c|c|c|c|c|c|c|c|c|c|c|c|c|c|c|c|c|c|}
\hline \multirow[t]{2}{*}{ No. } & \multicolumn{2}{|l|}{$\mathbf{0}$} & \multicolumn{2}{|l|}{$\mathbf{a}$} & \multicolumn{2}{|l|}{ b } & \multicolumn{2}{|l|}{ c } & \multicolumn{2}{|l|}{ d } & \multicolumn{2}{|l|}{ A } & \multicolumn{2}{|l|}{ B } & \multicolumn{2}{|l|}{ C } & \multicolumn{2}{|l|}{ D } \\
\hline & $\mathbf{w}$ & d & $\mathbf{w}$ & d & $\mathbf{w}$ & d & $\mathbf{w}$ & d & $\mathbf{w}$ & d & $\mathbf{w}$ & d & $\mathbf{w}$ & d & $\mathbf{w}$ & d & $\mathbf{w}$ & d \\
\hline$\overline{\mathrm{i}}$ & 2 & 2 & 2 & 3 & 2 & 3-4 & 2 & 3 & 2 & 3 & 2 & 3 & $2-3$ & 3-4 & 2 & 3 & $2-3$ & 4 \\
\hline ii & 2 & 2 & 2 & 3 & 2 & 3 & 2 & 3 & 2 & 3 & 2 & 3 & 2 & 3 & 2 & 3 & $2-3$ & 3-4 \\
\hline iii & 2 & $2-3$ & $2-3$ & 3 & $2-3$ & 3 & $2-3$ & 3 & $2-3$ & 3 & $2-3$ & 3 & 3 & 3 & $2-3$ & 3 & 3 & 3-4 \\
\hline iv & 2 & 3 & $2-3$ & 3 & 2-3 & 3 & $2-3$ & 3 & $2-3$ & 3 & 3 & 3-4 & 3 & 3-4 & $2-3$ & 3 & 3 & 3-4 \\
\hline $\mathrm{V}$ & 2 & 2 & 2 & 3 & $2-3$ & $3-4$ & 2 & 3 & $2-3$ & 3-4 & $2-3$ & 3-4 & $2-3$ & 3-4 & $2-3$ & 3-4 & $2-3$ & 3-4 \\
\hline vi & 2 & 2-3 & 2 & 3 & 2-3 & 3-4 & $2-3$ & 3-4 & 2-3 & 3-4 & 3 & 3-4 & 3 & 3-4 & $2-3$ & 3-4 & 3 & 4 \\
\hline vii & 2 & 2 & 2 & 3 & $2-3$ & 3 & 2 & 3 & 2 & 3 & 2 & 3 & $2-3$ & 3 & 2 & 3 & $2-3$ & 3-4 \\
\hline viii & 2 & 2 & 2 & 2 & 2 & 2 & 2 & 2 & 2 & 2 & 2 & 2 & 2 & $2-3$ & 2 & 2 & $2-3$ & 3 \\
\hline$M$ & 2 & 2 & 2 & $2-3$ & 2 & 3 & 2 & 3 & 2 & 3 & 2 & 3 & $2-3$ & 3 & 2 & 3 & $2-3$ & $3-4$ \\
\hline$S D$ & 0 & 0.46 & 0.35 & 0.35 & 0.52 & 0.54 & 0.46 & 0.54 & 0.46 & 0.54 & 0.52 & 0.64 & 0.52 & 0.52 & 0.46 & 0.54 & 0.46 & 0.52 \\
\hline$V$ & 0 & 0.21 & 0.13 & 0.13 & 0.27 & 0.29 & 0.21 & 0.29 & 0.21 & 0.29 & 0.27 & 0.41 & 0.27 & 0.27 & 0.21 & 0.29 & 0.21 & 0.27 \\
\hline
\end{tabular}

i) Madder; ii) RS Wood ; iii) Barberry ; iv) Annatto ; v) Myrobalan ; vi)

Pomogranate

vii) Onion ; viii) Grape

3.2 KES-F data of natural colored silk

The KES-F data of natural colored silk fabrics are shown in Tables $2 b$ with respect to the standard descriptions given in the Table 2a. The details regarding the hand values with respect to smoothness, stiffness and fullness of the natural colored silk fabric are presented in the Table $2 \mathrm{~b}$. When the combination of finishing treatment using recovered protein with other natural finishing sources increases, in different combined form the smoothness and fullness increases and stiffness decreases correspondingly after coloration with natural sources compared with those of the untreated natural colored silk fabric as evidenced in the Table $2 \mathrm{~b}$.

Table 2a. KES-F data of natural colored silk

\begin{tabular}{|c|}
\hline Common description (Unit) $^{\mid \text {Tensile }^{\text {a }}}$ \\
\hline 1) LT - Linearity of Load/extension curve (None) \\
\hline 2) WT - Tensile energy (N/m) \\
\hline 3) RT - Tensile resilience (\%) \\
\hline
\end{tabular}




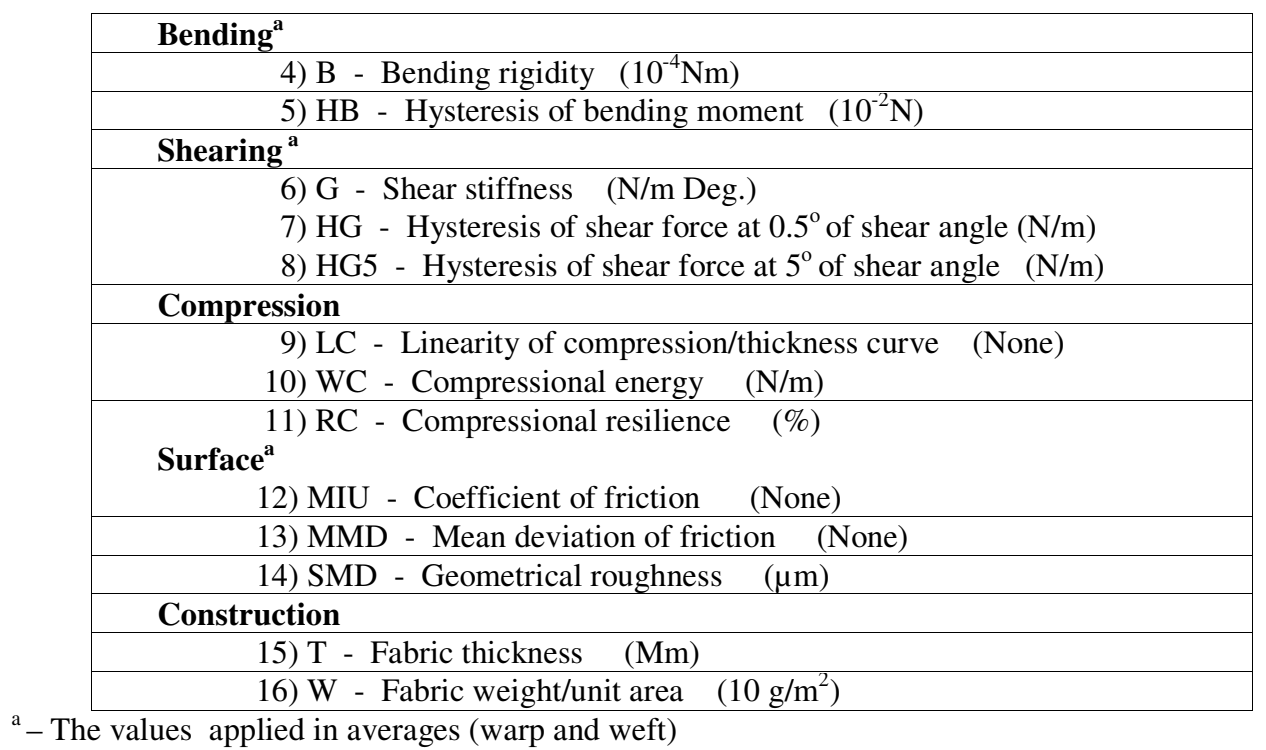

a - The values applied in averages (warp and weft)

Table $2 \mathrm{~b}$. Hand value of the natural colored silk

\begin{tabular}{|c|c|c|c|c|c|c|c|c|c|c|c|c|}
\hline \multirow{2}{*}{$\begin{array}{l}\text { Treat } \\
\text { ments }\end{array}$} & \multicolumn{12}{|c|}{ Hand value of the natural colored silk [Smoothness] } \\
\hline & $\begin{array}{l}\text { No } \\
\text { Dye }\end{array}$ & $\begin{array}{l}\text { Mad } \\
\text { der }\end{array}$ & RSW & $\begin{array}{l}\text { Bar } \\
\text { berry }\end{array}$ & $\begin{array}{l}\text { Ann } \\
\text { atto }\end{array}$ & $\begin{array}{l}\text { Myro } \\
\text { balan }\end{array}$ & $\begin{array}{l}\text { Pomogr } \\
\text { anate }\end{array}$ & $\begin{array}{l}\text { On } \\
\text { ion }\end{array}$ & $\begin{array}{l}\text { Gr } \\
\text { ape }\end{array}$ & $M$ & $S D$ & $\boldsymbol{V}$ \\
\hline $\mathbf{0}$ & 5.9 & 6.2 & 6.1 & 6.2 & 6.3 & 6.3 & 6.2 & 6.1 & 6.1 & 6.2 & 0.08 & 0.006 \\
\hline $\mathbf{a}$ & 7.2 & 7.5 & 7.5 & 7.6 & 7.6 & 7.7 & 7.7 & 7.5 & 7.5 & 7.6 & 0.08 & 0.007 \\
\hline b & 7.5 & 7.9 & 7.8 & 7.8 & 7.8 & 7.9 & 8.0 & 7.8 & 7.8 & 7.8 & 0.13 & 0.02 \\
\hline c & 7.3 & 7.6 & 7.7 & 7.5 & 7.4 & 7.6 & 7.7 & 7.6 & 7.6 & 7.6 & 0.09 & 0.009 \\
\hline d & 7.4 & 7.8 & 7.7 & 7.7 & 7.8 & 7.7 & 7.8 & 7.7 & 7.7 & 7.7 & 0.05 & 0.002 \\
\hline A & 7.5 & 8.1 & 8.2 & 8.3 & 8.3 & 8.4 & 8.4 & 8.3 & 8.2 & 8.3 & 0.09 & 0.009 \\
\hline B & 7.6 & 8.3 & 8.4 & 8.5 & 8.4 & 8.6 & 8.6 & 8.4 & 8.3 & 8.4 & 0.11 & 0.012 \\
\hline $\mathbf{C}$ & 7.5 & 8.0 & 8.1 & 8.0 & 8.0 & 8.1 & 8.2 & 8.1 & 8.0 & 8.1 & 0.07 & 0.005 \\
\hline D & 7.8 & 8.6 & 8.8 & 8.8 & 8.7 & 8.8 & 8.8 & 8.7 & 8.6 & 8.7 & 0.08 & 0.007 \\
\hline \multirow{2}{*}{$\begin{array}{l}\text { Treat } \\
\text { ments }\end{array}$} & \multicolumn{12}{|c|}{ Hand value of the natural colored silk [Stiffness] } \\
\hline & $\begin{array}{l}\text { No } \\
\text { Dye }\end{array}$ & $\begin{array}{l}\text { Mad } \\
\text { der }\end{array}$ & RSW & $\begin{array}{l}\text { Bar } \\
\text { berry }\end{array}$ & $\begin{array}{l}\text { Ann } \\
\text { atto }\end{array}$ & $\begin{array}{l}\text { Myrob } \\
\text { alan }\end{array}$ & $\begin{array}{l}\text { Pomogr } \\
\text { anate }\end{array}$ & $\begin{array}{l}\text { On } \\
\text { ion }\end{array}$ & $\begin{array}{l}\text { Gra } \\
\text { pe }\end{array}$ & $M$ & $S D$ & $\boldsymbol{V}$ \\
\hline 0 & 7.4 & 7.2 & 7.2 & 7.3 & 7.1 & 7.2 & 7.2 & 7.1 & 7.1 & 7.2 & 0.07 & 0.004 \\
\hline $\mathbf{a}$ & 7.0 & 6.7 & 6.8 & 6.7 & 6.8 & 6.7 & 6.8 & 6.7 & 6.8 & 6.8 & 0.05 & 0.003 \\
\hline b & 6.6 & 6.3 & 6.4 & 6.2 & 6.2 & 6.3 & 6.3 & 6.2 & 6.3 & 6.3 & 0.07 & 0.005 \\
\hline c & 6.9 & 6.5 & 6.7 & 6.5 & 6.6 & 6.6 & 6.7 & 6.6 & 6.7 & 6.6 & 0.08 & 0.006 \\
\hline d & 6.8 & 6.4 & 6.6 & 6.3 & 6.4 & 6.5 & 6.5 & 6.4 & 6.5 & 6.5 & 0.09 & 0.008 \\
\hline $\mathbf{A}$ & 6.4 & 6.1 & 6.1 & 6.0 & 6.0 & 6.1 & 6.2 & 6.1 & 6.0 & 6.1 & 0.07 & 0.004 \\
\hline B & 6.3 & 6.0 & 6.0 & 6.0 & 5.9 & 5.9 & 6.0 & 6.0 & 5.9 & 6.0 & 0.05 & 0.002 \\
\hline C & 6.5 & 6.2 & 6.2 & 6.1 & 6.1 & 6.2 & 6.2 & 6.1 & 6.2 & 6.2 & 0.05 & 0.002 \\
\hline D & 6.1 & 5.9 & 5.8 & 5.9 & 5.7 & 5.7 & 5.8 & 5.8 & 5.7 & 5.8 & 0.08 & 0.006 \\
\hline Treat & \multicolumn{12}{|c|}{ Hand value of the natural colored silk [Fullness] } \\
\hline
\end{tabular}




\begin{tabular}{|l|l|l|l|l|l|l|l|l|l|l|l|l|} 
ments & $\begin{array}{l}\text { No } \\
\text { Dye }\end{array}$ & $\begin{array}{l}\text { Mad } \\
\text { der }\end{array}$ & RSW & $\begin{array}{l}\text { Bar } \\
\text { berry }\end{array}$ & $\begin{array}{l}\text { Ann } \\
\text { atto }\end{array}$ & $\begin{array}{l}\text { Myrob } \\
\text { alan }\end{array}$ & $\begin{array}{l}\text { Pomogr } \\
\text { anate }\end{array}$ & $\begin{array}{l}\text { On } \\
\text { ion }\end{array}$ & $\begin{array}{l}\text { Gra } \\
\text { pe }\end{array}$ & $\boldsymbol{M}$ & $\boldsymbol{S D}$ & $\boldsymbol{V}$ \\
\hline 0 & 6.5 & 6.7 & 6.7 & 6.8 & 6.7 & 6.7 & 6.8 & 6.7 & 6.8 & 6.7 & 0.05 & 0.002 \\
$\mathrm{a}$ & 7.3 & 7.6 & 7.5 & 7.8 & 7.7 & 7.6 & 7.7 & 7.5 & 7.7 & 7.6 & 0.09 & 0.009 \\
\hline $\mathrm{b}$ & 7.9 & 8.2 & 8.1 & 8.2 & 8.3 & 8.2 & 8.3 & 8.3 & 8.0 & 8.2 & 0.10 & 0.01 \\
\hline $\mathrm{c}$ & 7.5 & 7.8 & 7.7 & 7.9 & 7.8 & 7.8 & 7.9 & 7.7 & 7.8 & 7.8 & 0.07 & 0.005 \\
\hline $\mathrm{d}$ & 7.7 & 8.0 & 7.9 & 8.1 & 8.0 & 8.0 & 8.1 & 8.0 & 7.9 & 8.0 & 0.07 & 0.005 \\
$\mathrm{~A}$ & 8.1 & 8.4 & 8.4 & 8.5 & 8.6 & 8.5 & 8.6 & 8.5 & 8.5 & 8.5 & 0.07 & 0.005 \\
\hline $\mathrm{B}$ & 8.2 & 8.5 & 8.5 & 8.6 & 8.7 & 8.7 & 8.8 & 8.7 & 8.7 & 8.7 & 0.10 & 0.01 \\
$\mathrm{C}$ & 8.0 & 8.3 & 8.3 & 8.4 & 8.4 & 8.3 & 8.4 & 8.4 & 8.3 & 8.4 & 0.05 & 0.003 \\
\hline $\mathrm{D}$ & 8.4 & 8.7 & 8.6 & 8.8 & 8.8 & 8.9 & 8.9 & 8.8 & 8.8 & 8.8 & 0.09 & 0.009 \\
\hline
\end{tabular}

3.3 Air-permeability and water-vapor-permeability of the natural colored silk fabrics

The values of air and water vapor permeability respectively of silk fabrics treated with selected finishing sources like aloevera, amla, nocchi and recovered protein in the intact as well as in the combined form and colored using the natural sources such as madder, red sandal wood, barberry, annatto, myrobalan, pomogranate, onion, and grape are shown in Table 3. From the Table 3, it is observed that there is a uniform trend in the air permeability and water vapor permeability behaviors as revealed by the silk fabrics after different treatments and colorations. The air permeability is in accordance with the behavior of the water vapor permeability of the silk fabric. In connection with that the air permeability and water vapor permeability values are maximum for silk fabrics treated with finishing type of natural sources such as aloevera, amla, nochi and recovered protein followed by colored with natural sources (D, B, A and C).

Table 3. Air-permeability and water-vapor-permeability of the natural colored silk

\begin{tabular}{|c|c|c|c|c|c|c|c|c|c|c|c|c|}
\hline \multirow{2}{*}{$\begin{array}{l}\text { Treat } \\
\text { ments }\end{array}$} & \multicolumn{12}{|c|}{ Air-permeability of the natural colored silk ( $1 / \mathrm{min})$} \\
\hline & $\begin{array}{l}\text { No } \\
\text { Dye }\end{array}$ & $\begin{array}{l}\text { Mad } \\
\text { der }\end{array}$ & RSW & $\begin{array}{l}\text { Bar } \\
\text { berry }\end{array}$ & $\begin{array}{l}\text { Ann } \\
\text { atto }\end{array}$ & $\begin{array}{l}\text { Myro } \\
\text { balan }\end{array}$ & $\begin{array}{l}\text { Pomogr } \\
\text { anate }\end{array}$ & $\begin{array}{l}\text { On } \\
\text { ion }\end{array}$ & $\begin{array}{l}\text { Gr } \\
\text { ape }\end{array}$ & $M$ & $S D$ & $\boldsymbol{V}$ \\
\hline 0 & 72 & 78 & 78 & 79 & 81 & 80 & 79 & 80 & 80 & 79.38 & 0.99 & 0.98 \\
\hline $\mathrm{a}$ & 75 & 85 & 86 & 86 & 88 & 86 & 86 & 88 & 87 & 86.5 & 1 & 1 \\
\hline $\mathrm{b}$ & 93 & 105 & 105 & 106 & 105 & 106 & 107 & 106 & 105 & 105.6 & 0.69 & 0.48 \\
\hline $\mathrm{c}$ & 82 & 93 & 94 & 93 & 94 & 93 & 94 & 95 & 94 & 93.8 & 0.66 & 0.44 \\
\hline $\mathrm{d}$ & 87 & 98 & 99 & 99 & 99 & 98 & 100 & 100 & 99 & 99 & 0.71 & 0.5 \\
\hline A & 98 & 113 & 114 & 114 & 115 & 113 & 115 & 116 & 115 & 114.4 & 0.99 & 0.98 \\
\hline $\mathrm{B}$ & 102 & 118 & 118 & 119 & 120 & 118 & 119 & 120 & 120 & 119 & 0.87 & 0.75 \\
\hline $\mathrm{C}$ & 95 & 109 & 109 & 110 & 110 & 109 & 111 & 111 & 110 & 109.9 & 0.78 & 0.61 \\
\hline $\mathrm{D}$ & 106 & 126 & 125 & 124 & 125 & 125 & 124 & 126 & 125 & 125 & 0.71 & 0.5 \\
\hline \multirow{2}{*}{$\begin{array}{l}\text { Treat } \\
\text { ments }\end{array}$} & \multicolumn{12}{|c|}{ Water-vapor-permeability of the natural colored silk (g/m²/day) } \\
\hline & $\begin{array}{l}\text { No } \\
\text { Dye }\end{array}$ & $\begin{array}{l}\text { Mad } \\
\text { der }\end{array}$ & RSW & $\begin{array}{l}\text { Bar } \\
\text { berry }\end{array}$ & $\begin{array}{l}\text { Ann } \\
\text { atto }\end{array}$ & $\begin{array}{l}\text { Myro } \\
\text { balan }\end{array}$ & $\begin{array}{l}\text { Pomogr } \\
\text { anate }\end{array}$ & $\begin{array}{l}\text { On } \\
\text { ion }\end{array}$ & $\begin{array}{l}\text { Gr } \\
\text { ape }\end{array}$ & $M$ & $S D$ & $V$ \\
\hline 0 & 1578 & 1612 & 1618 & 1620 & 1620 & 1618 & 1620 & 1620 & 1619 & 1618.4 & 2.55 & 6.48 \\
\hline
\end{tabular}




\begin{tabular}{|l|l|l|l|l|l|l|l|l|l|l|l|l|} 
a & 1655 & 1690 & 1696 & 1698 & 1698 & 1697 & 1695 & 1697 & 1698 & 1696.1 & 2.52 & 6.36 \\
\hline b & 1685 & 1725 & 1727 & 1729 & 1730 & 1732 & 1728 & 1731 & 1730 & 1729 & 2.12 & 4.5 \\
\hline c & 1667 & 1705 & 1708 & 1710 & 1712 & 1711 & 1708 & 1712 & 1710 & 1709.5 & 2.24 & 5 \\
\hline d & 1675 & 1713 & 1716 & 1720 & 1719 & 1720 & 1717 & 1720 & 1719 & 1718 & 2.35 & 5.5 \\
\hline A & 1697 & 1740 & 1739 & 1743 & 1742 & 1744 & 1742 & 1743 & 1742 & 1741.9 & 1.54 & 2.36 \\
\hline B & 1704 & 1748 & 1747 & 1750 & 1751 & 1751 & 1750 & 1751 & 1750 & 1749.8 & 1.39 & 1.94 \\
C & 1690 & 1732 & 1732 & 1735 & 1735 & 1736 & 1734 & 1736 & 1736 & 1734.5 & 1.58 & 2.5 \\
\hline D & 1714 & 1760 & 1760 & 1762 & 1762 & 1763 & 1762 & 1763 & 1762 & 1761.8 & 1.09 & 1.19 \\
\hline
\end{tabular}

\subsection{Uv-protection-factor-rating of the natural colored silk}

The uv protection factor rating of the dyed (madder, red sandal wood, barberry, annatto, myrobalan, pomegranate, onion, and grape) silk fabrics treated with protein, aloevera, amla and nochi in different combinations was carried out by UV visible spectrophotometer and the data are given in Table 4. From the Table 4, it is seen that except the untreated one all the other natural colored and finished silk fabrics show very good to excellent uv protection factor values. In general, there is a maximum uv protection factor of the natural colored silk fabrics subjected with all the combinations (D) followed by the others (B, A, C, b, d, c, a and 0). Textiles are able to offer intrinsically uv protection as they have their inherent good protection against intense radiation from the sun if provided with the selective materials and constructions. UPF (uv protection factor) could be attributed far above for those of the sustained sunscreens (sun blocks). The intent for this method in the involvement of textiles for sun protection is verily highly demanded. Based on this concept, in order to assess the degree of protection to solar ultraviolet radiation of textile materials, in vitro test methods are probably used.

Table 4. Uv-protection-factor-rating of the natural colored silk

\begin{tabular}{|c|c|c|c|c|c|c|c|c|c|c|c|}
\hline \multirow[b]{2}{*}{$\begin{array}{l}\text { Treat } \\
\text { ments }\end{array}$} & \multicolumn{11}{|c|}{ Uv-protection-factor-rating of the natural colored silk } \\
\hline & $\begin{array}{l}\text { Madd } \\
\text { er }\end{array}$ & RSW & $\begin{array}{l}\text { berr } \\
\mathbf{y}\end{array}$ & $\begin{array}{l}\text { Ann } \\
\text { atto }\end{array}$ & $\begin{array}{l}\text { Myr } \\
\text { o } \\
\text { bala }\end{array}$ & $\begin{array}{l}\text { Pomog } \\
\text { ranate }\end{array}$ & $\begin{array}{l}\text { On } \\
\text { ion }\end{array}$ & $\begin{array}{l}\text { Gr } \\
\text { ape }\end{array}$ & $M$ & $S D$ & $\boldsymbol{V}$ \\
\hline 0 & 22 & 20 & 21 & 20 & 22 & 21 & 22 & 20 & 21.00 & 0.87 & 0.75 \\
\hline $\mathrm{a}$ & 26 & 25 & 26 & 25 & 26 & 26 & 27 & 25 & 25.75 & 0.66 & 0.44 \\
\hline $\mathrm{b}$ & 38 & 39 & \multirow{2}{*}{$\begin{array}{l}38 \\
34\end{array}$} & 37 & 39 & 38 & 39 & 38 & 38.25 & 0.66 & 0.44 \\
\hline $\mathrm{c}$ & 32 & 33 & & 33 & 35 & 34 & 36 & 35 & 34.00 & 1.23 & 1.50 \\
\hline $\mathrm{d}$ & 34 & 34 & 35 & 34 & 36 & 35 & 37 & 36 & 35.13 & 1.05 & 1.11 \\
\hline A & 42 & 41 & 41 & 40 & 40 & 41 & 41 & 40 & 40.75 & 0.66 & 0.44 \\
\hline B & 42 & 42 & 42 & 43 & 42 & 43 & 42 & 41 & 42.13 & 0.60 & 0.36 \\
\hline $\mathrm{C}$ & 40 & 40 & 39 & 38 & 39 & 39 & 40 & 39 & 39.25 & 0.66 & 0.44 \\
\hline $\mathrm{D}$ & 43 & 43 & 42 & 44 & 44 & 43 & 44 & 43 & 43.25 & 0.66 & 0.44 \\
\hline \multicolumn{12}{|c|}{ Uv-protection-factor-rating for the textiles (Standard chart) } \\
\hline \multicolumn{4}{|c|}{ Uv-protection-factor-rating } & \multicolumn{3}{|c|}{ Category of protection } & \multicolumn{5}{|c|}{ Uv radiation blocked $(\%)$} \\
\hline \multicolumn{4}{|l|}{$15-24$} & \multicolumn{3}{|l|}{ GOOD } & \multicolumn{5}{|c|}{$93.3-95.9$} \\
\hline \multicolumn{4}{|l|}{$25-39$} & \multicolumn{3}{|c|}{ VERY GOOD } & \multicolumn{5}{|c|}{$96-97.4$} \\
\hline
\end{tabular}


3.5 $\mathrm{X}$ ray diffraction analysis of the natural colored silk

The $\mathrm{X}$ ray diffraction micrographs of the representative silk samples such as; the degummed silk fabric (1a), the silk fabric colored with red sandal (RSW) wood (1b), the protein (recovered sericin) treated and red sandal wood colored silk fabric (1c), the recovered sericin (with aloevera) treated and red sandal wood colored silk fabric (1d), the recovered sericin (with aloevera and nochi) treated and red sandal wood colored silk fabric (1e), and the recovered sericin (with aloevera, amla and nochi) treated and red sandal wood colored silk fabric (1f) are given in Figure 1. The respective combined effect of $\mathrm{X}$ ray diffraction spectrogram of the natural colored silk is presented in the Figure 2. It is clear from the Figure 1 , that there is a clear change in the sharpness of the peak in the XRD graphs. Also it is evident from the Figure 1 that the change is more obvious when the finishing source applications are increased on the silk fabrics. Subsequently, the silk fabrics applied with recovered sericin, aloevera and nochi (1e) and recovered sericin, aloevera, amla and nochi (1f) followed by coloring with red sandal wood indicate the change in a clear manner in the $\mathrm{X}$ ray diffractions micrographs.

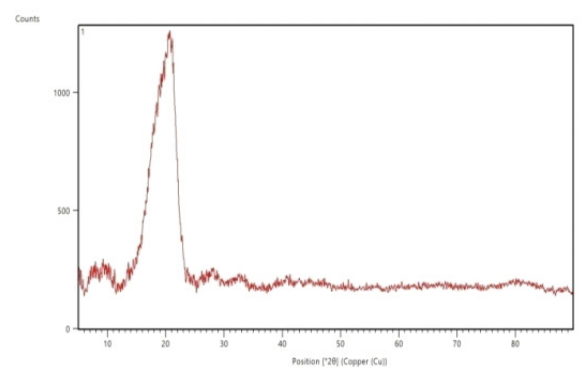

1a. Degummed silk fabric

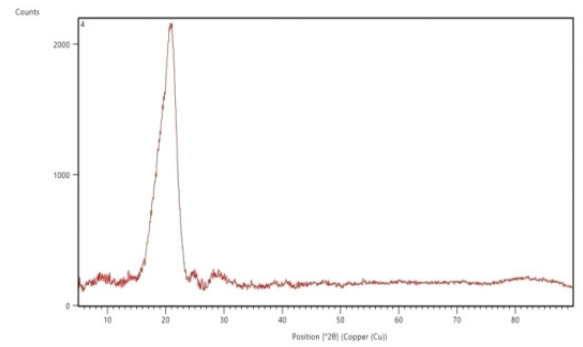

1c. Protein treated and RSW and

colored silk fabric

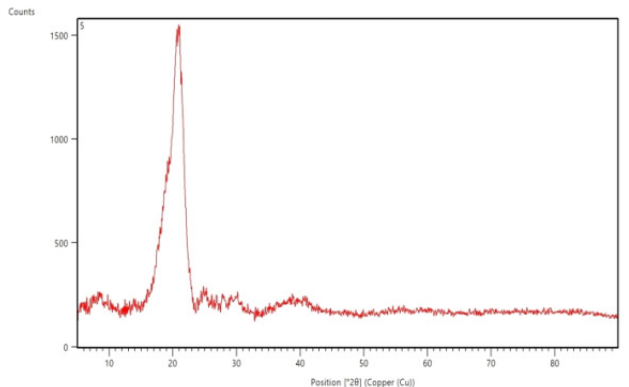

1b. Silk fabric colored with RSW

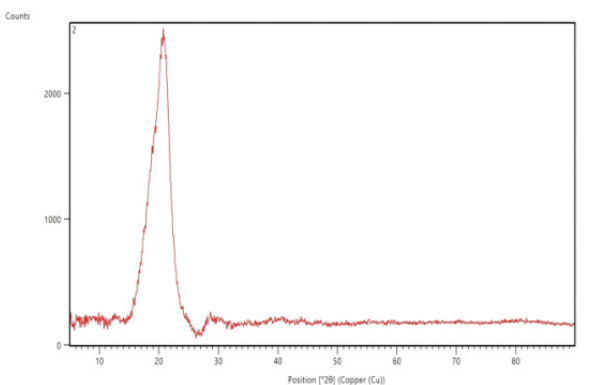

1d. Protein (with aloevera) treated

RSW colored silk fabric 


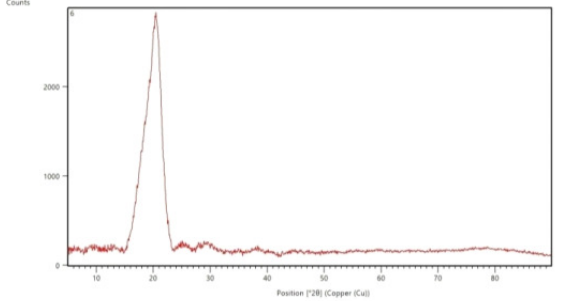

1e. Protein (with aloevera and nochi) treated and RSW colored silk fabric

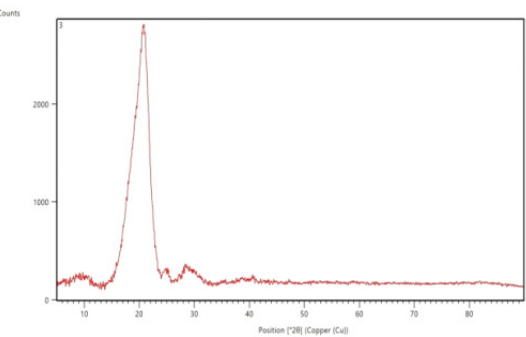

1f. Protein (with aloevera, amla and nochi) treated and RSW colored silk fabric

Fig 1. X ray diffraction spectrogram of the natural colored silk
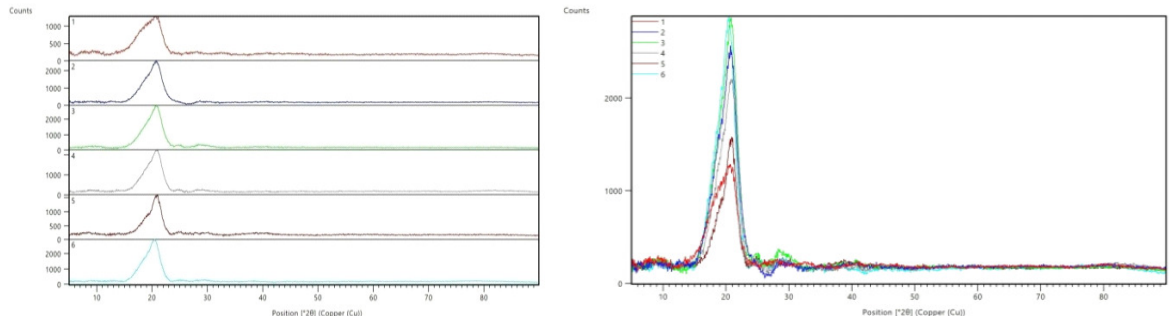

Fig 2. Combined effect of $X$ ray diffraction spectrogram of the natural colored silk

3.6 Scanning electron microscope analysis of the natural colored silk

The silk fabrics with the representative scanning electron microscope images are only shown in Figure 3. The degummed silk fabric (3a), the protein (recovered sericin) treated and red sandal wood (RSW) colored silk fabric (3b), the recovered sericin (with aloevera and nochi) treated and red sandal wood colored silk fabric (3c), and the recovered sericin (with aloevera, amla and nochi) treated and red sandal wood colored silk fabric (3d) are the respective samples given in the Figure 3. It is evident from these images that when the finishing source treatments (recovered sericin, aloevera, nochi and amla) increased, the effect on the silk fabric also increases. The enhanced finishing effect is visible in the scanning electron microscope images in the case of the silk fabric treated with recovered sericin, aloevera and nochi (3c); and recovered sericin, aloevera, amla and nochi (3d) followed by coloring with red sandal wood. 


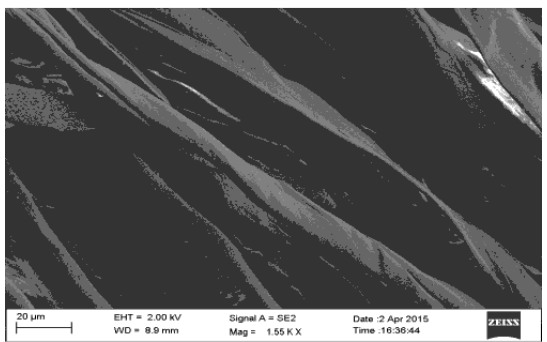

3a. Degummed silk fabric

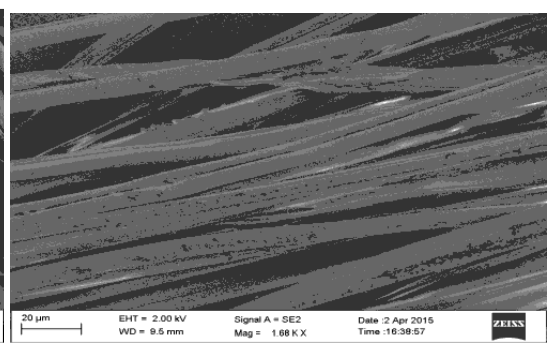

3b. Protein treated and RSW colored silk

fabric

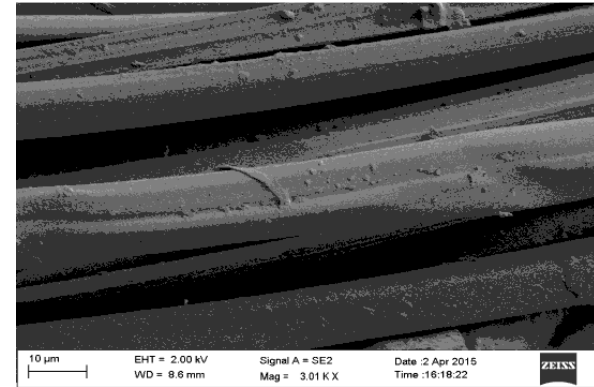

3c. Protein (with aloevera and nochi) treated and RSW colored silk fabric

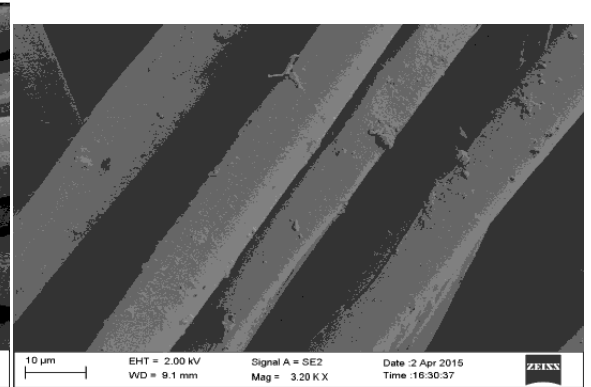

3d. Protein (with aloevera, amla and nochi) treated and RSW colored silk fabric

Fig 3. Scanning electron microscope micrographs of the natural colored silk

\section{CONCLUSIONS}

The colorimetric data for the coloration are increased with the increase of treatment using the finishing type of natural sources such as aloevera, amla, nochi and recovered sericin on silk fabrics and colored with natural sources. The increase of colorimetric data is maximum on the silk fabric treated with recovered sericin along with aloevera, amla and nochi followed by colored with natural sources. The fastness property rating for the natural colored silk fabric subjected with the recovered sericin together with aloevera, amla, and nochi in different combined form is in the good to very good trend. There is a moderate to good fastness property in the silk fabric applied using the recovered sericin with aloevera, amla, and nochi and colored with natural sources.

There is a very good air-permeability in accordance with the behavior of the watervapor-permeability in the silk fabric. Obviously, the air permeability and water vapor permeability values are higher for the silk fabrics subjected with finishing type of natural sources such as aloevera, amla, nochi and recovered sericin followed by coloration with natural sources.

The surface hand value characters of the silk fabrics applied using the recovered sericin with the aloevera, amla, and nochi and colored with the natural sources are in the good trend.

The $\mathrm{x}$ ray diffraction micrograms give the clear indication of the positive changes in the silk fabrics subjected with recovered sericin, aloevera and nochi; and recovered sericin, aloevera, amla and nochi followed by the coloration. The scanning electron microscope 
images show the increased finishing effect in the case of the silk fabric subjected with recovered sericin, aloevera and nochi; and recovered sericin, aloevera, amla and nochi followed by the coloration.

\section{ACKNOWLEDGEMENT}

The authors wish to thank the Management and the Principal, PSG College of Technology, Coimbatore for given the permission and providing the necessary infrastructure. Thanks are also due to The Head, Department of Applied Science for the kind help in department laboratory supports.

\section{References}

[1] Akai H, Imai T and Tsubouchi K 1987 Fine structural changes of liquid silk in the silkgland during the spinning stage of Bombyx mori larvae J. seric. Sci. Jpn 56131

[2] Inoue S, Kanda T, Imamura M, Quan GX, Kojma K, Tanaka H et al 2005 A fibroin secretion deficient silkworm mutant, Nd-sD, provides an efficient system for producing recombinant proteins Insect Biochem. Mol. Biol 3551

[3] Gregory HA, Diaz F, Caroline J, Tora C, Rebecca LH, Jingsong C, Helen L, John R and David LK 2003 Silk based biomaterials Biomaterials $24 \quad 401$

[4] Asakura T, Yamare T, Nakasawa Y, Kamada T and Ando K 2001 Structure of Bombyx mori silk fibroin before spinning in solid state studied with wide angle $\mathrm{X}$ - ray scattering and 13 cross polarization/magic angle spinning NMR Biopolymers $58 \quad 521$

[5] Komatsu K 1975 Studies on dissolution behaviors and structural characteristic of silk Sericin Bull. Sericult. Exp. Sta 26135

[6] Shimizu M 2000 Structural basis of silk fibre; in Structure of silk yarn vol-I, biological and physical aspects Hojo, N (ed.) (Oxford \& IBH Publ. Co. Pvt. Ltd New Delhi) 7

[7] Yamada M 1978 Amino acid composition of the sericin extracted from cocoon of the mulberry wild silkworm, Bombyx mori and its species specificity J. Sericult. Sci. Japan 47108

[8] Konishi T 2000 Structure of fibroin $-\alpha$ in Structure of silk yarn Hojo, N. (ed) (Oxford and IBH pubn. Co. Pvt. Ltd New Delhi) 267

[9] Matta A, Migliaresi C, Faccioni F, Torricelli P, Fini M and Giardino R 2004 Fibroin hydrogels for biomedical applications, preparation, characterization and in vitro cell culture studies $\mathrm{J}$. Biomater. Sci. Polym 15851

[10] Robson RM 1985 Silk composition, structure and properties; in Hand book of fibre Science and Technology:vol-IV Lewin M and pearce EM(ed) (Mercel. Dekker Inc New York) 649

[11] Devaraju N 2002 Degumming of silk - A review Colourage 4943

[12] Gulrajani ML1988 Degumming of silk; in Silk dyeing printing and finishing, Dept. Text Tech (Indian Inst. of Tech. New Delhi) 63

[13] Tsukada M 1983 Structure of silk sericins removed from wild silk by boiling in water J. Sericult. Sci. Japan $52 \quad 296$

[14] Komatsu K 1980 Recent advances in sericin research J. Sericult. Sci. Japan 69457

[15] Jihad R 2014 Dyeing of Silk Using Natural Dyes Extracted From Local Plants Internl. J. Sci Eng Res. 5809

[16] Win ZM and Swe MM 2008 Purification of the Natural Dyestuff Extracted from Mango Bark for the Application on protein fibers World Acad, Sci Eng Technol 22536

[17] Trotman ER 1984 Dyeing and Chemical Technology of Textile Fibers 6th edition, (Eds) Edward Arnold (London, UK) 187

[18] Mohanty BC, Chandramauti KV and Naik HD 1987 Natural dyeing process of India (Published by Calico Museum of Textiles India)

[19] Broadbent AD 2001 Basic principles of textile coloration, Society of Dyers and Colorists (Published by the Soc. Dyers Colourists England) 
[20] Mourad S, Emmel P and Simon K 2001 Extending Kubelka-Munks theory with lateral light scattering Internl. Conf. Digital Printing Technl. (Florida USA)

[21] Agarwal D, Sen K and Gulrajani, ML 1997 Dyeing of silk with bifunctional reactive dyes: the relationship between exhaustion and fixation J. Soc. Dyers and Colourists 113174

[22] AATCC test method-61-1996, 2003 Color fastness to laundering: Home and communication accelerated AATCC Technical manual (Research Triangle Park USA)

[23] AATCC test method 16-1998, 2003 Color fastness to light AATCC Technical manual (Research Triangle Park USA)

[24] AATCC test method 8-2007, 2007 Color fastness to crocking AATCC Technical manual (Research Triangle Park USA)

[25] AATCC test method 135-1985, 2003 Color measurement of textiles: Instrumental AATCC Technical manual (Research Triangle Park USA)

[26] Niwa M 1991 Objective measurement of fabric mechanical property and quality its application to textile and clothing manufacturing Internl. J. Clothing Sci Tech 37

[27] ASTM - D737-2012 Standard test methods for air permeability of textile fabrics ASTM International 100 Barr Harbor Drive, PO Box C700 (West Conshohocken PA 19428-2959, USA)

[28] ASTM - E96-2000 Standard test methods for water vapor transmission of materials ASTM International 100 Barr Harbor Drive, PO Box C700 (West Conshohocken, PA 19428-2959, USA) 